\title{
Strategyproof Linear Regression in High Dimensions
}

\author{
YILING CHEN, Harvard University, USA \\ CHARA PODIMATA, Harvard University, USA \\ ARIEL D. PROCACCIA, Carnegie Mellon University, USA \\ NISARG SHAH, University of Toronto, Canada
}

\begin{abstract}
This paper is part of an emerging line of work at the intersection of machine learning and mechanism design, which aims to avoid noise in training data by correctly aligning the incentives of data sources. Specifically, we focus on the ubiquitous problem of linear regression, where strategyproof mechanisms have previously been identified in two dimensions. In our setting, agents have single-peaked preferences and can manipulate only their response variables. Our main contribution is the discovery of a family of group strategyproof linear regression mechanisms in any number of dimensions, which we call generalized resistant hyperplane mechanisms. The game-theoretic properties of these mechanisms - and, in fact, their very existence - are established through a connection to a discrete version of the Ham Sandwich Theorem.
\end{abstract}

\section{INTRODUCTION}

Designing machine learning algorithms that are robust to noise in training data is a topic of intense research. A large body of work addresses stochastic noise [20,29]. On the other extreme, another branch of the literature focuses on adversarial noise $[6,12,25]$, that is, errors are introduced by an adversary with the explicit purpose of sabotaging the algorithm. The latter approach is often too pessimistic, and generally leads to negative results.

More recently, some researchers have taken a game-theoretic viewpoint; it suggests a model of strategic noise that can be seen as occupying the middle ground of noise models. Specifically, training data is provided by strategic sources - hereinafter agents - that may intentionally introduce errors to maximize their own benefit. Compared to adversarial noise, the advantage of this model (when its underlying assumptions hold true) is that, if we aligned the agents' incentives correctly, it would be possible to obtain uncontaminated data. From this viewpoint, the ideal is the design of learning algorithms that in addition to being statistically efficient, are strategyproof, i.e., where supplying pristine data is a dominant strategy for each agent.

We subscribe to this agenda, and advance it in the context of the ubiquitous problem of linear regression, i.e., fitting a hyperplane through given data. We consider agents who can manipulate

\footnotetext{
This work was partially supported by the National Science Foundation under grants CCF-1718549, IIS-1350598, IIS-1714140, CCF-1525932, and CCF-1733556; by the Office of Naval Research under grants N00014-16-1-3075 and N00014-17-1-2428; by a Sloan Research Fellowship and a Guggenheim Fellowship; and by the Natural Sciences and Engineering Research Council of Canada (NSERC) under the Discovery Grants program.

Authors' addresses: Yiling Chen, Harvard University, Cambridge, MA, 02138, USA, yiling@seas.harvard.edu; Chara Podimata, Harvard University, Cambridge, MA, 02138, USA, podimata@g.harvard.edu; Ariel D. Procaccia, Carnegie Mellon University, Pittsburgh, PA, 15213, USA, arielpro@cs.cmu.edu; Nisarg Shah, University of Toronto, Toronto, ON, M5S 3G8, Canada, nisarg@cs.toronto.edu.
}

\footnotetext{
Permission to make digital or hard copies of all or part of this work for personal or classroom use is granted without fee provided that copies are not made or distributed for profit or commercial advantage and that copies bear this notice and the full citation on the first page. Copyrights for components of this work owned by others than the author(s) must be honored Abstracting with credit is permitted. To copy otherwise, or republish, to post on servers or to redistribute to lists, requires prior specific permission and/or a fee. Request permissions from permissions@acm.org.
}

(c) 2018 Copyright held by the owner/author(s). Publication rights licensed to ACM.

ACM EC'18, fune 18-22, 2018, Ithaca, NY, USA. ACM ISBN 978-1-4503-5829-3/18/06 ..\$15.00

https://doi.org/10.1145/3219166.3219175 
their dependent variables in order to minimize their vertical distance from the output hyperplane, and design strategyproof regression mechanisms without payments.

When does this type of strategic regression problem arise? Dekel et al. [15] give the real-world example of the global fashion chain Zara, whose distribution process relies on regression [9]. Specifically, the demand for each product at each store is predicted based on historical data, as well as information provided by store managers. Since the supply of popular items is limited, store managers may strategically manipulate requested quantities so that the output of the regression process would better fit their needs, and, indeed, there is ample evidence that many of them have done so [10]. More generally, as discussed in detail by Perote and Perote-Peña [36], this type of setting is relevant whenever "data could come from surveys composed by agents interested in not being perceived as real outliers if the estimation results could be used in the future to change the economic situation of the agents that generate the sample."

\subsection{Our Model and Results}

A bit more formally, we study a linear regression setting in which the task is to fit a hyperplane through data points $\left(\boldsymbol{x}_{i}, y_{i}\right)$ for $i \in\{1, \ldots, n\}$, where $\boldsymbol{x}_{i} \in \mathbb{R}^{d}$ are the independent variables and $y_{i} \in \mathbb{R}$ is the dependent variable. Following Dekel et al. [15] and Perote and Perote-Peña [36], we assume that the independent variables are public information, but dependent variable $y_{i}$ is held privately by agent $i$. A mechanism elicits the private information of the agents, and returns a hyperplane represented by vector $\boldsymbol{\beta}=\left(\boldsymbol{\beta}_{1}, \beta_{0}\right) \in \mathbb{R}^{d+1}$. Under this outcome, the residual for agent $i$ is $r_{i}=y_{i}-\boldsymbol{\beta}_{1}^{T} \boldsymbol{x}_{i}-\beta_{0}$, and, loosely speaking, agents wish to minimize $\left|r_{i}\right|$ (see Section 2 for a precise description of agent preferences).

Our starting point is the work of Dekel et al. [15], who show that empirical risk minimization (ERM) with the $L_{1}$ loss (in short, $L_{1}$-ERM), coupled with a specific tie-breaking rule, is group strategyproof, that is, no coalition of agents can be weakly better off by misreporting. We extend this result and show that replacing the $L_{1}$ loss by a weighted $L_{1}$ loss and adding convex regularization to the risk function preserves group strategyproofness. But this still gives a relatively restricted family of strategyproof mechanisms, and we seek a broader understanding of what is possible in our setting.

To that end, we look to the work of Perote and Perote-Peña [36], who focus on the twodimensional case (known as simple linear regression), i.e., fitting a line through points on a plane. They propose a wide family of strategyproof mechanisms, which they call clockwise repeated median (CRM) mechanisms. These mechanisms are parametrized by two subsets of agents $S$ and $S^{\prime}$. Perote and Perote-Peña [36] establish conditions on $S$ and $S^{\prime}$ under which they claim that CRM mechanisms are strategyproof. We identify a mistake in this result, present counterexamples showing violation of strategyproofness under their conditions, and identify three stricter conditions under which we can recover strategyproofness - in fact, we prove group strategyproofness. Under one of our conditions, CRM mechanisms coincide with a family of mechanisms from the statistics literature known as resistant line mechanisms [24]. Our work therefore establishes the group strategyproofness of these mechanisms.

Our main result is that we generalize the CRM family to higher dimensions, thereby justifying the title of this paper. We introduce the family of generalized resistant hyperplane (GRH) mechanisms, which, to the best of our knowledge, is the first extension of resistant line mechanisms beyond the plane. In $d+1$ dimensions, GRH mechanisms are parametrized by $d+1$ subsets of agents. Through a surprising connection to the literature on the Ham Sandwich Theorem, we find a condition on the subsets under which GRH mechanisms are group strategyproof. Strikingly, our proof of this general group strategyproofness result in any number of dimensions is much shorter than the 
(incorrect) proof of Perote and Perote-Peña [36] for the strategyproofness of CRM mechanisms in two dimensions.

We also study a property called impartiality, which is stricter than strategyproofness. We establish the existence of a wide family of impartial mechanisms, which, unlike our generalized $L_{1}$-ERM and generalized resistant hyperplane mechanisms, are strategyproof but not group strategyproof (except for constant functions). Building upon the work of Moulin [33], we also provide two non-constructive characterizations of strategyproof mechanisms for linear regression.

Strategyproofness is not the sole desideratum; constant functions (e.g., the flat hyperplane $y=0$ ) are strategyproof but not necessarily desirable. We would also like the mechanism to have good statistical efficiency. For that, we compare (families of) strategyproof mechanisms in terms of their approximation of the optimal squared loss, leveraging our characterization. Most importantly, we establish a lower bound of 2 on the approximation ratio of any strategyproof mechanism, which means that any mechanism that is even close to ordinary least squares regression must be manipulable.

\subsection{Related Work}

As discussed above, our work is most closely related to that of Perote and Perote-Peña [36] and Dekel et al. [15]. Here we try to give a broader picture of the state of research on machine learning algorithms that are robust to strategic noise. This research can be categorized using three key axes: (i) manipulable information, (ii) goal of the agents, and (iii) use of payments and incentive guarantees.

On the first axis, like us, most papers assume that independent variables (or feature vectors in the language of classification) are public information, and dependent variables (labels) are private, manipulable information [15, 32, 35, 36], though some papers also design algorithms robust to strategic feature vectors [16, 21]. Meir et al. [32] provide strong positive results for designing strategyproof classifiers when there are either only two classifiers, or the agents are interested in a shared set of input points. On the other hand, Hardt et al. [21] study the problem of constructing classifiers that are robust to agents strategically misreporting their feature vector, in order to trick the algorithm into misclassifying them. Their setting is modeled as a one-shot Stackelberg game. The more recent work of Dong et al. [16] models the same problem in an online setting; they provide guarantees that ensure that the problem is convex, and, therefore, they are able to derive a computationally efficient learning algorithm that has diminishing Stackelberg regret.

On the second axis, one line of research focuses on agents motivated by privacy concerns, with a tradeoff between accuracy and privacy [7,13]; another focuses on agents who want the algorithm to make accurate assessment on their own sample, even if this reduces the overall accuracy. This form of strategic manipulation has been studied for estimation [8], classification [30-32], and regression $[15,36]$ problems. Our problem falls squarely into the second category.

Finally, on the third axis, various papers differ on whether monetary payments to agents are allowed [7], and on how strongly to guarantee truthful reporting: the stronger strategyproofness requirement [32, 35, 36] versus the weaker Bayes-Nash incentive compatibility [13, 23]. Our work falls into the literature of mechanism design without money; we study linear regression mechanisms that enforce strategyproofness without paying the agents, or asking the agents to pay.

\section{MODEL}

Let $[k] \triangleq\{1, \ldots, k\}$ be the set of first $k$ natural numbers, and $\overline{\mathbb{R}}=\mathbb{R} \cup\{-\infty, \infty\}$ be the extended real line. Given numbers $t_{1}, \ldots, t_{k} \in \overline{\mathbb{R}}$, let $\min \left(t_{1}, \ldots, t_{k}\right)$ denote the smallest value, and $\min ^{j}\left(t_{1}, \ldots, t_{k}\right)$ denote the $j^{\text {th }}$ smallest value. Let $\operatorname{med}\left(t_{1}, \ldots, t_{k}\right)$ denote their median: when $k$ is odd, this is equal to 
$\min ^{(k+1) / 2}\left(t_{1}, \ldots, t_{k}\right)$, but when $k$ is even, this could be either $\min ^{k / 2}\left(t_{1}, \ldots, t_{k}\right)$ (the "left median") or $\min ^{k / 2+1}\left(t_{1}, \ldots, t_{k}\right)$ (the "right median"). ${ }^{1}$

Our work focuses on the problem of linear regression, i.e., fitting a hyperplane through given data. Let $N=[n]$. We are given a collection of data points $\mathcal{D}=\left(\boldsymbol{x}_{i}, y_{i}\right)_{i \in N}$, where $\boldsymbol{x}_{i} \in \mathbb{R}^{d}$ and $y_{i} \in \mathbb{R}$ are called the independent and dependent variables of point $i$, respectively. Let $\overline{\boldsymbol{x}_{i}}=\left(\boldsymbol{x}_{i}, 1\right)$. Our goal is to find a vector $\boldsymbol{\beta}=\left(\boldsymbol{\beta}_{1}, \beta_{0}\right) \in \mathbb{R}^{d+1}$ such that $\boldsymbol{\beta}^{T} \overline{\boldsymbol{x}_{i}}=\boldsymbol{\beta}_{1}^{T} \boldsymbol{x}_{i}+\beta_{0}$ is a good approximation of $y_{i}$ for each $i \in N$. The quantity $r_{i}=y_{i}-\boldsymbol{\beta}^{T} \overline{\boldsymbol{x}_{i}}$ is called the residual of point $i$.

Strategic setting. We study a setting in which each data point $p_{i}=\left(\boldsymbol{x}_{i}, y_{i}\right)$ is provided by a strategic agent $i$. We also denote the set of agents by $N$. Following Perote and Perote-Peña [36] and Dekel et al. [15], we assume that the independent variables $\boldsymbol{x}=\left(\boldsymbol{x}_{i}\right)_{i \in N}$ constitute public information, which the agents cannot manipulate. Each agent $i$ holds the dependent variable $y_{i}$ as private information, and may report a different value $\widetilde{y}_{i}$ in order to receive a more preferred outcome. Thus, the principal observes the reported data points $\widetilde{\mathcal{D}}=\left(\boldsymbol{x}_{i}, \widetilde{y}_{i}\right)_{i \in N}$. Let us denote $\boldsymbol{y}=\left(y_{i}\right)_{i \in N}$ and $\widetilde{\boldsymbol{y}}=\left(\widetilde{y}_{i}\right)_{i \in N}$.

Mechanisms. Because the agents cannot change $\boldsymbol{x}$, we can effectively treat it as fixed. A mechanism for linear regression $M^{x}$ is therefore defined for given public information $x$, takes as input reported private information $\tilde{\boldsymbol{y}}$, and returns a vector $\boldsymbol{\beta}$. We omit $\boldsymbol{x}$ when it is clear from the context.

Agent preferences. When a mechanism returns $\boldsymbol{\beta}$, we say that the outcome for agent $i$ is $\widehat{y}_{i}(\boldsymbol{\beta})=$ $\boldsymbol{\beta}^{T} \boldsymbol{x}_{i}$. We omit $\boldsymbol{\beta}$ when it is clear from the context. The agent only cares about her own outcome $\widehat{y}_{i}$, and would like it to be as close to $y_{i}$ as possible. Formally, we assume that agent $i$ has single-peaked preferences $[3,33]$ over $\widehat{y}_{i}$ with peak at $y_{i}$. We represent the weak preference relation by $\succcurlyeq_{i}$ and the strict preference relation by $>_{i}$. Formally, for all $a, b \in \mathbb{R}, y_{i}>a \geq b$ or $y_{i}<a \leq b$ must imply $y_{i}>_{i} a \succcurlyeq_{i} b$.

Game-theoretic desiderata. Our goal is to prevent agents from misreporting their private information. The game theory literature offers a strong desideratum under which agents have no incentive to misreport even if they have know what the other agents would report.

Definition 2.1 (Strategyproofness). A mechanism $M^{x}$ is called strategyproof (SP) if each agent weakly prefers truthfully reporting her private information to misreporting it, regardless of the reports of the other agents. Formally, for each $i \in N, y_{i} \in \mathbb{R}$, and $\widetilde{\boldsymbol{y}} \in \mathbb{R}^{n}$, we need $\widehat{y}_{i}\left(M^{x}\left(y_{i}, \widetilde{\boldsymbol{y}}_{-i}\right)\right) \succcurlyeq_{i}$ $\widehat{y_{i}}\left(M^{x}(\widetilde{\boldsymbol{y}})\right)$. Note that this must hold for any possible single-peaked preferences the agent may have.

While no individual agent can benefit from misreporting under a strategyproof mechanism, a group of agents may still be able to collude, and benefit by simultaneously misreporting. This can be prevented by imposing a stronger desideratum.

Definition 2.2 (Group Strategyproofness). A mechanism $M^{x}$ is called group strategyproof (GSP) if no coalition of agents can simultaneously misreport in a way that no agent in the coalition is strictly worse off and some agent in the coalition is strictly better off, irrespective of the reports of the other agents. Formally, for each $S \subseteq N, \boldsymbol{y}_{S}=\left(y_{i}\right)_{i \in S} \in \mathbb{R}^{|S|}$, and $\widetilde{\boldsymbol{y}} \in \mathbb{R}^{n}$, it should not be the case that $\widehat{y}_{i}\left(M^{x}(\widetilde{\boldsymbol{y}})\right) \succcurlyeq_{i} \widehat{y}_{i}\left(M^{x}\left(\boldsymbol{y}_{S}, \widetilde{\boldsymbol{y}}_{N \backslash S}\right)\right)$ for every $i \in S$, and the preference is strict for at least one $i \in S$.

\footnotetext{
${ }^{1}$ This is different from the standard definition, which takes the average of the left and right medians, but necessary to ensure incentive guarantees.
} 
The game theory literature also considers a weaker notion of group strategyproofness in which not all the agents in a manipulating coalition should be strictly better off. We do not consider this notion because our group strategyproof mechanisms are able to satisfy the stronger notion.

Note that we do not assume that the data points are generated by an underlying statistical process. Our results are independent of how the data points were generated.

\section{FAMILIES OF STRATEGYPROOF MECHANISMS}

In this section, we analyze families of (group) strategyproof mechanisms for linear regression. Our results generalize existing families of mechanisms, and propose novel families.

\subsection{Empirical Risk Minimization with the $L_{1}$ Loss}

Consider a single dimensional setting, in which each agent $i$ has a private value $y_{i}$, reports a possibly different value $\widetilde{y}_{i}$, and the mechanism returns a single value $\widehat{y}$. Each agent $i$ has single-peaked preferences over $\widehat{y}$ with peak at $y_{i}$. This corresponds to the special case of our setting in which $\boldsymbol{x}_{i}=\boldsymbol{x}_{j}$ for all $i, j \in N$, or alternatively, the dimension $d=0$. In this setting, it has long been known that choosing the median of the reported values achieves group strategyproofness [17]. It can be shown that the median minimizes the sum of absolute $\left(L_{1}\right)$ losses with respect to the reports, i.e., given $\boldsymbol{y}$, it chooses $\arg \min _{y \in \mathbb{R}} \sum_{i=1}^{n}\left|y-y_{i}\right|$, with an appropriate tie-breaking when $n$ is even. In the machine learning terminology, the median is the empirical risk minimizer (ERM) with the $L_{1}$ loss.

Inspired by this, Dekel et al. [15] study ERM with the $L_{1}$ loss in a more general regression setting, and show that it remains group strategyproof. Specifically, they focus on finding a (potentially non-linear) regression function $f: \mathbb{R}^{d} \rightarrow \mathbb{R}$ from a given convex set $\mathcal{F}$. Given $\mathcal{D}=\left(\boldsymbol{x}_{i}, y_{i}\right)_{i \in N}$, define the empirical $L_{1}$ risk of a regression function $f \in \mathcal{F}$ as $\widehat{R}(f, \mathcal{D})=\sum_{i \in N}\left|y_{i}-f\left(\boldsymbol{x}_{i}\right)\right|$. Let $\|\cdot\|: \mathcal{F} \rightarrow \mathbb{R}$ be a strictly convex function. They show that minimizing the empirical $L_{1}$ risk, and breaking ties among the optimal solutions by minimizing $\|\cdot\|$ is group strategyproof. We refer to this mechanism by $L_{1}$-ERM ${ }^{2}$. For linear regression, this approach is known by various names in the literature, such as Least Absolute Deviations (LAD), Minimum Sum of Absolute Errors (MSAE), or Least Absolute Value (LAV). The tie-breaking step is crucially required because the empirical $L_{1}$ risk may have multiple minimizers.

We present a generalization of their mechanism while retaining group strategyproofness. In particular, we extend the objective function $\widehat{R}$ in two ways: i) we allow a weighted $L_{1}$ loss, in which the loss of each agent $i$ is multiplied by a weight $w_{i}^{x}$, and ii) we allow adding a convex regularizer $h: \mathcal{F} \rightarrow \mathbb{R}$. Note that regularization is widely used in machine learning to prevent ERM from overfitting. Our generalization, which we term generalized $L_{1}-E R M$, is presented as Algorithm 1 . While we are only interested in linear regression, we note that generalized $L_{1}$-ERM works for the general regression setting of Dekel et al. [15].

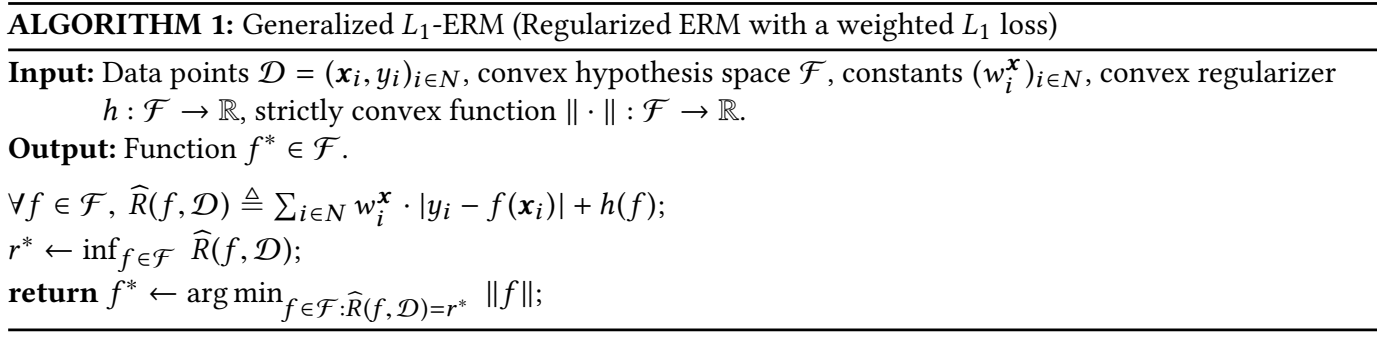

\footnotetext{
${ }^{2}$ For a formal description of the algorithm, we refer the interested reader to the full version of our paper (available on the authors' webpages).
} 
THEOREM 3.1. Generalized $L_{1}-E R M$ is a group strategyproof regression mechanism.

Our proof, presented in the full version for completeness, essentially mirrors the proof of Dekel et al. [15]; we identify three steps in their proof where they use the structure of the risk function $\widehat{R}$, and observe that these steps follow through with our more general risk function.

There are several potential advantages of generalized $L_{1}$-ERM over the vanilla $L_{1}$-ERM. First, generalized $L_{1}$-ERM allows eliminating the tie-breaking step if the new risk function is guaranteed to have a unique minimizer. For instance, adding a strictly convex regularizer would achieve this.

Second, for the aforementioned single dimensional setting, Moulin [33] proved that every strategyproof $^{3}$ and anonymous ${ }^{4}$ mechanism is a generalized median: for every $\alpha_{1}, \ldots, \alpha_{n+1} \in \overline{\mathbb{R}}$, the corresponding generalized median returns med $\left\{y_{1}, \ldots, y_{n}, \alpha_{1}, \ldots, \alpha_{n+1}\right\}$. Here, $\left\{\alpha_{j}\right\}_{j \in[n+1]}$ are called "phantoms". We can alternatively view this as returning $\arg \min _{y \in \mathbb{R}} \sum_{i \in[n]}\left|y-y_{i}\right|+h(y)$, where $h(y)=\sum_{j \in[n+1] \text { s.t. } \alpha_{j} \in \mathbb{R}}\left|y-\alpha_{j}\right|+\left(k_{-\infty}-k_{\infty}\right) \cdot y$, and for $t \in\{-\infty, \infty\}, k_{t}=\left|\left\{j: \alpha_{j}=t\right\}\right|{ }^{5}$ Since $h(y)$ is a convex function, we can view it as a regularizer in our generalized $L_{1}$-ERM. Hence, for the single dimensional setting, generalized $L_{1}$-ERM covers all generalized medians. In contrast, $L_{1}$-ERM reduces to a specific mechanism in this family, the median.

Finally, algorithms that add convex regularization to $L_{1}$-ERM have been studied in the machine learning literature [41, 42]; our generalization establishes group strategyproofness of these algorithms.

We also note that in the statistics literature, the vanilla $L_{1}$-ERM is treated as a member of the more general family of quantile regression mechanisms [27], which, given $q \in[0,1]$, minimize the following empirical risk function:

$$
\widehat{R}_{q}(f, \mathcal{D})=\sum_{i \in N: y_{i} \geq f\left(\boldsymbol{x}_{i}\right)} q \cdot\left|y_{i}-f\left(\boldsymbol{x}_{i}\right)\right|+\sum_{i \in N: y_{i}<f\left(\boldsymbol{x}_{i}\right)}(1-q) \cdot\left|y_{i}-f\left(\boldsymbol{x}_{i}\right)\right| .
$$

$L_{1}$-ERM corresponds to the choice of $q=0.5$. In the one-dimensional setting, other values of $q$ correspond to different quantiles (i.e., correspond to $\min ^{k}$ for various $k$ ), and thus induce strategyproof mechanisms. One might wonder if quantile regression remains strategyproof in higher dimensions. We answer this negatively by providing an example in the full version, in which the quantile regression mechanism for $q=0.4$ is shown to violate strategyproofness. It is an interesting question to discover a strategyproof version of quantiles for linear regression.

\subsection{Generalized Resistant Hyperplane Mechanisms}

In this section, we introduce a novel family of strategyproof mechanisms for linear regression. Our family extends the known family of resistant line mechanisms from the statistics literature [24], which were only defined for simple linear regression $(d=1)$, to higher dimensions. We first take a slight detour through a previous approach in the literature.

3.2.1 A Detour Through Clockwise Repeated Median Mechanisms. Perote and Perote-Peña [36] introduced a novel family of mechanisms, which they termed Clockwise Repeated Median (CRM) mechanisms. CRM mechanisms are only defined for the special case of simple linear regression, i.e., for fitting a straight line through a set of points on a plane. In describing these mechanisms, we use scalar notations where possible. For instance, we use $x_{i}$ to denote the $\mathrm{x}$-coordinate of agent $i$, and

\footnotetext{
${ }^{3}$ Moulin [33] shows that for the single dimensional setting, strategyproofness is equivalent to group strategyproofness.

${ }^{4} \mathrm{~A}$ mechanism is anonymous if permuting the reports of the agents does not change the output of the mechanism. This is a reasonable desideratum in the single dimensional setting due to the absence of public information that distinguishes agents naturally.

${ }^{5}$ When all phantoms are finite, $h(y)=\sum_{j \in[n+1]}\left|y-\alpha_{j}\right|$. The term $\left|y-\alpha_{j}\right|$ has derivative 1 when $y>\alpha_{j}$, and -1 when $y<\alpha_{j}$. For $\alpha_{j}=-\infty$ (resp. $\infty$ ), we can mimic this effect by adding a different term whose derivative is always -1 (resp. 1 ).
} 
$\beta_{1}$ to denote the slope of the regression line. For CRM mechanisms to be well defined, we also need to assume that the set of points is "admissible".

Definition 3.2 (Admissible Set). A collection of data points $\mathcal{D}=\left(x_{i}, y_{i}\right)_{i \in N}$ is called admissible if $x_{i} \neq x_{j}$ for all distinct $i, j \in N$.

The CRM family is parametrized by two subsets of agents, $S, S^{\prime} \subseteq N$. These subsets must be chosen based on the public information $\boldsymbol{x}$, and therefore can be treated as fixed. Informally, given $S, S^{\prime} \subseteq N$, the $\left(S, S^{\prime}\right)$-CRM mechanism first computes the median clockwise angle (CWA), defined below, from each point $i \in S$ to points in $S^{\prime}$. Then, it chooses the point $i^{*} \in S$ whose median CWA is the median of the median CWAs from all points in $S$. If the median CWA from point $i^{*}$ is towards point $j^{*} \in S^{\prime}$, then the mechanism returns the straight line passing through points $i^{*}$ and $j^{*}$. Formally, the mechanism is defined as follows. Perote and Perote-Peña [36] established the equivalence of this formal definition and the aforementioned informal description.

Definition 3.3 (CRM Mechanisms). Define the clockwise angle (CWA) from $\left(x_{i}, y_{i}\right)$ to $\left(x_{j}, y_{j}\right)$ as:

$$
\operatorname{CWA}\left(\left(x_{i}, y_{i}\right),\left(x_{j}, y_{j}\right)\right)=\pi+\operatorname{sign}\left(x_{j}-x_{i}\right) \cdot \frac{\pi}{2}+\operatorname{sign}\left(\frac{y_{j}-y_{i}}{x_{j}-x_{i}}\right)\left|\arctan \left(\frac{y_{j}-y_{i}}{x_{j}-x_{i}}\right)\right| \text {. }
$$

Given $\mathcal{D}=\left(x_{i}, y_{i}\right)_{i \in N}$ and $S, S^{\prime} \subseteq N$, let the directing angle be defined as:

$$
\operatorname{DA}\left(S, S^{\prime}\right)=\operatorname{med}_{i \in S} \operatorname{med}_{j \in S^{\prime}: j \neq i} \operatorname{CWA}\left(\left(x_{i}, y_{i}\right),\left(x_{j}, y_{j}\right)\right) .
$$

Then, the $\left(S, S^{\prime}\right)$-CRM mechanism returns the line $\boldsymbol{\beta}=\left(\beta_{1}, \beta_{0}\right)$ given by:

$$
\begin{aligned}
& \beta_{1}=\tan \left[\operatorname{DA}\left(S, S^{\prime}\right)-\pi-\frac{\pi}{2} \cdot \operatorname{sign}\left(\operatorname{DA}\left(S, S^{\prime}\right)-\pi\right)\right], \\
& \beta_{0}=\underset{i \in S}{\operatorname{med}}\left(y_{i}-\beta_{1} \cdot x_{i}\right) .
\end{aligned}
$$

First, we notice that the definition of the CRM family uses three medians: two to define the directing angle $D A\left(S, S^{\prime}\right)$, and one to define the $y$-intercept $\beta_{0}$. Each median, when taken over an even number of values, can be the left median or the right median. While Perote and Perote-Peña [36] do not mention how these choices should be made, it is easy to check that in order to achieve the desired incentive properties, these choices cannot be made independently of each other. Later, we present a generalization which captures the different feasible choices in a simpler form.

Perote and Perote-Peña [36] claimed that the $\left(S, S^{\prime}\right)$-CRM mechanism is strategyproof when $S \subseteq S^{\prime}$ or $S \cap S^{\prime}=\emptyset$, and provided an involved, geometric proof. However, we have identified a mistake in their proof. In fact, we have found two counterexamples, one with $S \subseteq S^{\prime}$ and one with $S \cap S^{\prime}=\emptyset$, for which the corresponding $\left(S, S^{\prime}\right)$-CRM mechanisms violate strategyproofness, thus disproving their claim. These counterexamples are presented in Figure 1,

Example 3.4 (Example with $S \cap S^{\prime}=\emptyset$.). This example is shown in Figure 1a. Points in filled dots are in $S$, while points in empty dots are in $S^{\prime}$. The coordinates of these points are as follows.

$$
S=\{(1,0),(3,1),(5,1.9)\}, S^{\prime}=\{(0,1),(2,2),(4,3)\} .
$$

Notice that $S \cap S^{\prime}=\emptyset$. Also, $|S|$ and $\left|S^{\prime}\right|$ are odd, alleviating the need to choose between left and right medians in the CRM definition.

When the agents truthfully report, one can check that CRM returns the line connecting points $(3,1)$ from $S$ and $(0,1)$ from $S^{\prime}$. This line is given by the equation $y=1$.

Suppose that the agent $i$ controlling the point at $x=4$ misreports $\widetilde{y}_{i}=1.8$ instead of $y_{i}=3$. The new point is depicted with a cross. One can check that this causes the CRM mechanism to switch to the dashed line $(y=0.1 \cdot x+1.4)$, which makes agent $i$ strictly better off, and violates strategyproofness. 


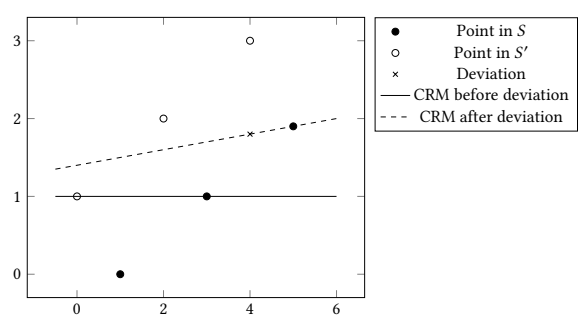

(a) $S \cap S^{\prime}=\emptyset$

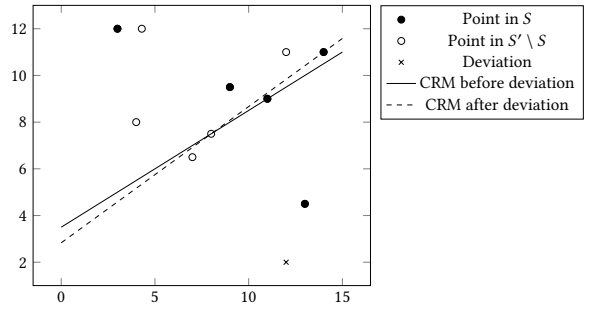

(b) $S \subseteq S^{\prime}$

Fig. 1. Counterexamples showing violation of strategyproofness of $\left(S, S^{\prime}\right)$-CRM mechanisms. Figure 1a shows a case with $S \cap S^{\prime}=\emptyset$, while Figure 1b shows a case with $S \subseteq S^{\prime}$.

Example 3.5 (Example with $S \subseteq S^{\prime}$.). This example is shown in Figure 1b. Points in $S$ (thus also in $S^{\prime}$ ) are depicted with filled dots, while points in $S^{\prime} \backslash S$ are depicted with empty dots. The coordinates of these points are as follows.

$S=\{(3,12),(9,9.5),(11,9),(13,4.5),(14,11)\}, S^{\prime}=S \cup\{(4,8),(4.3,12),(7,6.5),(8,7.5),(12,11)\}$.

Notice that $S \subseteq S^{\prime}$. Further, $|S|$ is odd, and $\left|S^{\prime}\right|$ is even (thus, for each $i \in S,\left|S^{\prime} \backslash\{i\}\right|$ is odd), once again eliminating the need to choose between the left and the right medians in the CRM definition.

When all points are reported truthfully, one can check that the CRM mechanism chooses the solid line $(3 y=2 x+8)$. Suppose now that agent $i$ with point $(12,11)$ reports $\widetilde{y}_{i}=0$, instead of $y_{i}=11$. Then, the CRM mechanism chooses the dashed line, which makes agent $i$ strictly better off, again violating strategyproofness.

Nevertheless, we have been able to identify a subset of the CRM family, for which we can establish strategyproofness (in fact, group strategyproofness). In particular, we replace $S \subseteq S^{\prime}$ with the more restrictive condition $S=S^{\prime}$, and for $S \cap S^{\prime}=\emptyset$, we either add $|S|=1$ or $\left|S^{\prime}\right|=1$, or replace it with a stricter condition that we define below.

Definition 3.6 (Separable Sets of Points in a Plane). Let $S, S^{\prime}$ be two sets of points in $\mathbb{R}^{2}$. We say that $S$ and $S^{\prime}$ are separable if $\max _{i \in S} x_{i}<\min _{j \in S^{\prime}} x_{j}$ or $\max _{j \in S^{\prime}} x_{j}<\min _{i \in S} x_{i}$. In other words, it should be possible to separate them by a vertical line.

Note that separability of $S$ and $S^{\prime}$ implies $S \cap S^{\prime}=\emptyset$. We now present a corrected version of the result of Perote and Perote-Peña [36], and claim the stronger guarantee of group strategyproofness. We do not present a proof as we later introduce a much broader family of mechanisms, and prove their group strategyproofness directly.

TheOREM 3.7. Given $S, S^{\prime} \subseteq N$, the $\left(S, S^{\prime}\right)$-CRM mechanism is group strategyproof if one of the following conditions holds.

(1) $S=S^{\prime}$.

(2) $S$ and $S^{\prime}$ are separable.

(3) $S \cap S^{\prime}=\emptyset$ and $\min \left(|S|,\left|S^{\prime}\right|\right)=1$.

The third condition partially resembles dictatorship as the agent in the singleton set is guaranteed to have zero residual (i.e., be on the regression line).

3.2.2 Generalized Resistant Line Mechanisms on a Plane. In this section, our goal is to introduce a novel family of group strategyproof mechanisms that include, as special cases, the mechanisms covered in the three cases of Theorem 3.7. Our starting point is the family of resistant line (RL) 
mechanisms from the statistics literature [24], which Perote and Perote-Peña [36] showed to be equivalent to the case of separable $S$ and $S^{\prime}$.

The standard formulation of the RL mechanism involves three sets $L, M, R \subseteq N$ such that $\max _{i \in L} x_{i}<\min _{i \in M} x_{i}$ and $\max _{i \in M} x_{i}<\min _{i \in R} x_{i}$, and returns a line $\boldsymbol{\beta}=\left(\beta_{1}, \beta_{0}\right)$ given by

$$
\operatorname{med}_{i \in L} y_{i}-\beta_{1} \cdot x_{i}-\beta_{0}=\operatorname{med}_{i \in R} y_{i}-\beta_{1} \cdot x_{i}-\beta_{0}=0 \text {. }
$$

That is, the line makes the median residuals in $L$ and $R$ zero. It is known that this equation yields a unique solution [24]. Perote and Perote-Peña [36] showed that this is identical to the $(L, R)$-CRM mechanism. Indeed, separability of $L$ and $R$ makes clockwise angles from points in $L$ to points in $R$ monotonic in (and thus replaceable by) slopes, yielding the following formulation for the $(L, R)$-CRM mechanism.

$$
\begin{aligned}
& \beta_{1}=\operatorname{med}_{i \in L} \operatorname{med}_{j \in R} \frac{y_{j}-y_{i}}{x_{j}-x_{i}}, \\
& \beta_{0}=\operatorname{med}_{i \in L} y_{i}-\beta_{1} x_{i}=\operatorname{med}_{j \in R} y_{j}-\beta_{1} x_{j} .
\end{aligned}
$$

The alternative definition of $\beta_{0}=\operatorname{med}_{j \in R}\left(y_{j}-\beta_{1} \cdot x_{j}\right)$ follows from the fact that if the line passes through $i^{*} \in L$, it is directed towards the point in $R$ which is at the median angle or slope, and thus bisects $R$ in addition to bisecting $L$.

Along with Theorem 3.7, this observation establishes group strategyproofness of all resistant line mechanisms. Two popular mechanisms from this family are the Brown-Mood mechanism [5], in which $L$ and $R$ each contain half of the points while $M$ is empty, and the Tukey mechanism [40], in which $L, M$, and $R$ each contain a third of the points.

Our next step is to extend this family. A natural idea is that instead of making the median residuals from $S$ and $S^{\prime}$ zero, we make the $k^{\text {th }}$ smallest residual in $S$ and the $\left(k^{\prime}\right)^{\text {th }}$ smallest residual in $S^{\prime}$ zero, for fixed $k \in[|S|]$ and $k^{\prime} \in\left[\left|S^{\prime}\right|\right]$.

Definition 3.8 (Generalized Resistant Line (GRL) Mechanisms). Given separable sets $S, S^{\prime} \subseteq N$, $k \in[|S|]$, and $k^{\prime} \in\left[\left|S^{\prime}\right|\right]$, the $\left(S, S^{\prime}, k, k^{\prime}\right)$-generalized resistant line (GRL) mechanism returns the line $\boldsymbol{\beta}=\left(\beta_{1}, \beta_{0}\right)$ given by

$$
\min _{i \in S}^{k} y_{i}-\beta_{1} x_{i}-\beta_{0}=\min _{j \in S^{\prime}}^{k^{\prime}} y_{j}-\beta_{1} x_{j}-\beta_{0}=0 .
$$

We show that these mechanisms are well defined (i.e., there is a unique solution to Equation (5)), and they are group strategyproof. Once again, we omit the proof because we later introduce an even broader family of mechanisms, for which we prove these results directly.

Theorem 3.9. For separable sets $S, S^{\prime} \subseteq N, k \in[|S|]$ and $k^{\prime} \in\left[\left|S^{\prime}\right|\right]$, the $\left(S, S^{\prime}, k, k^{\prime}\right)$-generalized resistant line mechanism is well defined and group strategyproof.

While it is clear that generalized resistant line mechanisms cover the second case of Theorem 3.7 (i.e., separable $S$ and $S^{\prime}$ ), we surprisingly find that they also cover the first case $\left(S=S^{\prime}\right)$ and the third case $\left(S \cap S^{\prime}=\emptyset\right.$ and $\left.\min \left(|S|,\left|S^{\prime}\right|\right)=1\right)$. That is, Theorem 3.9 strictly generalizes Theorem 3.7. The proof of the next result is in the full version.

LemMA 3.10. The $\left(S, S^{\prime}\right)$-CRM mechanism is a generalized resistant line mechanism when (1) $S=S^{\prime}$, (2) $S$ and $S^{\prime}$ are separable, or (3) $S \cap S^{\prime}=\emptyset$ and $\min \left(|S|,\left|S^{\prime}\right|\right)=1$.

3.2.3 Generalized Resistant Hyperplane Mechanisms in High Dimensions. Surprisingly, the statistics literature does not offer an extension of resistant line mechanisms to higher dimensions. In our efforts to do so, we quickly realized that this is a non-trivial task. In two dimensions, a generalized resistant line mechanism takes two subsets of data points separable by a vertical line, and returns the regression line which makes prescribed percentiles of residuals in each set zero. 
In $d+1$ dimensions (recall that $\boldsymbol{x}_{i} \in \mathbb{R}^{d}$ and $y_{i} \in \mathbb{R}$ ), it seems natural to take $d+1$ "separable" subsets of data points, and return the regression hyperplane which makes prescribed percentiles of residuals in each set zero. However, the separability condition must now ensure existence of a unique hyperplane with this property, even if we ignore our game-theoretic desiderata.

In resolving this issue, we make a connection to the literature on the Ham Sandwich Theorem and its generalizations. Hereinafter, given a hyperplane $H$, we denote by $\mathrm{H}^{+}$and $\mathrm{H}^{-}$its positive and negative closed half-spaces, respectively. A basic version of the ham sandwich theorem due to Stone and Tukey [38] states that given $k$ continuous measures $\mu_{1}, \ldots, \mu_{k}$ on $\mathbb{R}^{k}$, there exists a hyperplane $H$ such that $\mu_{i}\left(H^{+}\right)=1 / 2$ for each $i \in[k]$. A discrete version of this result due to Elton and Hill [18] states that given $k$ finite sets $S_{1}, \ldots, S_{k} \subseteq \mathbb{R}^{k}$, there exists a hyperplane $H$ such that for each $i \in[k], H$ "bisects" $S_{i}$ and $H \cap S_{i} \neq \emptyset$. Here, we say that a hyperplane $H$ bisects a set of points $S$ if each closed half-space of $H$ contains at least $\lceil|S| / 2\rceil$ points.

For linear regression, this implies that given $S_{1}, \ldots, S_{d+1} \subseteq \mathcal{D}$, there exists a "resistant hyperplane" which makes the median residual from $S_{t}$ zero, for each $t \in[d+1]$. While this seems like a natural generalization of resistant line mechanisms, it is easy to check that such a hyperplane is not always unique, even in two dimensions. Further, if the median is replaced by other percentiles, the existence is no longer guaranteed. ${ }^{6}$

Steiger and Zhao [37] provide a generalization that almost perfectly fits our needs. They show that under certain conditions on $S_{1}, \ldots, S_{d+1}$, there exists a unique hyperplane $H$ which contains a given number of points from each set in its negative closed half-space. This discrete result builds upon previous continuous variants $[1,4]$. We first define a condition they require, which also plays a key role in our result.

Definition 3.11 (Well Separable Sets [26]). Given $t \in[k+1]$, finite sets $S_{1}, \ldots, S_{t}$ of points in $\mathbb{R}^{k}$ are called well separable if for all disjoint $I, J \subseteq[t]$, there exists a hyperplane $H$ such that $S_{i} \subset H^{+} \backslash H$ for each $i \in I$ and $S_{j} \subset H^{-} \backslash H$ for each $j \in J$, i.e., $H$ separates $\cup_{i \in I} S_{i}$ from $\cup_{j \in J} S_{j}$ by putting them in different open half-spaces.

Well separable sets are sometimes called affinely independent sets [4]. Well separability is equivalent to various other conditions $[4,37]$. In what follows, $\operatorname{Conv}(\cdot)$ denotes the convex hull.

Proposition 3.12. For $t \in[k+1]$, finite sets $S_{1}, \ldots, S_{t} \subset \mathbb{R}^{k}$ are well separable if and only if:

(1) For all choices of $\left(x_{i} \in \operatorname{Conv}\left(S_{i}\right)\right)_{i \in[t]}$, the affine hull of $x_{1}, \ldots, x_{t}$ is a $(t-1)$-dimensional flat.

(2) No $(t-2)$-dimensional flat has a nonempty intersection with $\operatorname{Conv}\left(S_{i}\right)$ for each $i \in[t]$.

(3) $\operatorname{Conv}\left(S_{1}\right), \ldots, \operatorname{Conv}\left(S_{t}\right)$ are well separable.

Steiger and Zhao [37] impose an additional condition, which we eliminate in our work.

Definition 3.13 (Weak General Position). Finite sets $S_{1}, \ldots, S_{k} \subset \mathbb{R}^{k}$ are said to have weak general position if for every choice of $\left(x_{i} \in S_{i}\right)_{i \in[k]}$, the affine hull of $x_{1}, \ldots, x_{k}$ is a $(k-1)$-dimensional flat which contains no other point of $\cup_{i \in[k]} S_{i}$.

THeOREM 3.14 ([37]). If finite sets $S_{1}, \ldots, S_{k} \subset \mathbb{R}^{k}$ are well separable and have weak general position, then given any choice of $k_{i} \in\left[\left|S_{i}\right|\right]$ for $i \in[k]$, there exists a unique hyperplane $H$ such that for each $i \in[k], H \cap S_{i} \neq \emptyset$ and $\left|H^{-} \cap S_{i}\right|=k_{i}$.

This result gives us almost what we want for linear regression in $\mathbb{R}^{d+1}$. Given a family of sets $S_{1}, \ldots, S_{d+1} \subseteq \mathcal{D}$ that are well separable and have weak general position, and $k_{t} \in\left[\left|S_{t}\right|\right]$ for $t \in[d+1]$, it ensures the existence of a unique hyperplane which makes the $k_{t}^{\text {th }}$ smallest residual in each set $S_{t}$ zero. However, it falls short of our requirements in two key aspects.

\footnotetext{
${ }^{6}$ Recall that even in two dimensions, we needed an additional condition on the sets $S$ and $S^{\prime}$ : separability by a vertical line.
} 
- Theorem 3.14 allows the assignment of points in $\mathcal{D}$ to sets $S_{1}, \ldots, S_{d+1}$ to depend on the private information $\boldsymbol{y}$. For strategyproofness, we need this assignment to be based solely on the public information $\boldsymbol{x}$. Recall that in two dimensions, we required sets $S$ and $S^{\prime}$ to be separable by a vertical line. We choose the $d+1$ sets so that they are well separable in the $d$-dimensional public information space, ${ }^{7}$ and establish group strategyproofness using a technical lemma, which may be of independent interest.

- While we only want to make the $k_{t}^{\text {th }}$ smallest residual in each $S_{t}$ zero, Steiger and Zhao [37] aim for something stronger: they want the number of points from each $S_{t}$ in the negative closed halfspace to be exactly $k_{t}$. This necessitates their weak general position assumption, which we relax.

We are now ready to present our results. They closely mirror, but do not make use of, the results of Steiger and Zhao [37]. We revert to using notation of our linear regression setting. Recall that a hyperplane $\boldsymbol{\beta}=\left(\boldsymbol{\beta}_{1}, \beta_{0}\right)$ passes through $\left(\boldsymbol{x}_{i}, \boldsymbol{\beta}^{T} \overline{\boldsymbol{x}_{i}}\right)$ for each $i \in N$, where $\overline{\boldsymbol{x}_{i}}=\left(\boldsymbol{x}_{i}, 1\right)$.

Definition 3.15. Given a family $\mathcal{S}=\left(S_{1}, \ldots, S_{k}\right)$ of nonempty, pairwise disjoint subsets of $N$, and a set of points $P=\left(p_{i}\right)_{i \in N}$, define the partition function $\mathcal{P}(P, \mathcal{S})=\left(P_{t}\right)_{t \in[k]}$, where $P_{t}=\left(p_{i}\right)_{i \in S_{t}}$ for each $t \in[k]$. That is, $\mathcal{P}(P, \mathcal{S})$ partitions the set of points $P$ based on index sets from $\mathcal{S}$.

Definition 3.16 (Publicly Separable Sets of Agents). We say that a family $\mathcal{S}=\left(S_{1}, \ldots, S_{d+1}\right)$ of nonempty, pairwise disjoint subsets of $N$ is publicly separable if $\mathcal{P}(\boldsymbol{x}, \mathcal{S})$ is well separable.

Definition 3.17 (Generalized Resistant Hyperplane (GRH) Mechanisms). Given a family $\mathcal{S}=$ $\left(S_{1}, \ldots, S_{d+1}\right)$ of publicly separable sets of agents, and $\boldsymbol{k}=\left(k_{1}, \ldots, k_{d+1}\right)$ with $k_{t} \in\left[\left|S_{t}\right|\right]$ for $t \in[d+1]$, the $(\mathcal{S}, \boldsymbol{k})$-generalized resistant hyperplane (GRH) mechanism returns a hyperplane $\boldsymbol{\beta}$ such that $\min _{i \in S_{t}}^{k_{t}}\left(r_{i} \triangleq y_{i}-\boldsymbol{\beta}^{T} \overline{\boldsymbol{x}_{i}}\right)=0$ for each $t \in[d+1]$. That is, it makes the $k_{t}^{\text {th }}$ smallest residual from every set $S_{t} \in \mathcal{S}$ zero.

We first need to establish that the GRH mechanisms are well defined, i.e., the hyperplane they seek is guaranteed to exist and be unique. To that end, we prove a useful technical lemma, which may be of independent interest.

Lemma 3.18 (Hyperplane Comparison Lemma). Given a family $\mathcal{S}=\left(S_{1}, \ldots, S_{d+1}\right)$ of publicly separable sets of agents, and two distinct hyperplanes $\boldsymbol{\beta}^{1}$ and $\boldsymbol{\beta}^{2}$ in $\mathbb{R}^{d+1}$, there exists a set $S_{t} \in \mathcal{S}$ such that either $\left(\boldsymbol{\beta}^{1}\right)^{T} \overline{\boldsymbol{x}_{i}}<\left(\boldsymbol{\beta}^{2}\right)^{T} \overline{\boldsymbol{x}_{i}}$ for all $i \in S_{t}$, or $\left(\boldsymbol{\beta}^{1}\right)^{T} \overline{\boldsymbol{x}_{i}}>\left(\boldsymbol{\beta}^{2}\right)^{T} \overline{\boldsymbol{x}_{i}}$ for all $i \in S_{t}$.

Proof. Consider the intersection of the two hyperplanes in $\mathbb{R}^{d+1}$, and let $W$ be its projection on $\mathbb{R}^{d}$ (the public information space). Note that $W$ is a $(d-1)$-dimensional hyperplane in $\mathbb{R}^{d}$. Given an open half-space of $W$ (say $W^{+}$), let $Z$ be the set of points $\mathbb{R}^{d+1}$ whose projection on $\mathbb{R}^{d}$ lies in $W^{+}$. Then, either $\left(\boldsymbol{\beta}^{1}\right)^{T} \overline{\boldsymbol{p}}>\left(\boldsymbol{\beta}^{2}\right)^{T} \overline{\boldsymbol{p}}$ for all $\boldsymbol{p} \in Z$, or $\left(\boldsymbol{\beta}^{1}\right)^{T} \overline{\boldsymbol{p}}<\left(\boldsymbol{\beta}^{2}\right)^{T} \overline{\boldsymbol{p}}$ for all $\boldsymbol{p} \in Z$, where $\overline{\boldsymbol{p}}=(\boldsymbol{p}, 1)$.

Let $\mathcal{P}(\boldsymbol{x}, \mathcal{S})=\left(X_{1}, \ldots, X_{d+1}\right)$. Because $\mathcal{S}$ is publicly separable, $X_{1}, \ldots, X_{d+1}$ are well separable. By Proposition 3.12, no $(d-1)$-dimensional flat has a nonempty intersection with $\operatorname{Conv}\left(X_{t}\right)$ for each $t \in[d+1]$. Because $W$ is a $(d-1)$-dimensional flat, there exists $t \in[d+1]$ such that $W$ does not intersect $\operatorname{Conv}\left(X_{t}\right)$, i.e., $X_{t}$ lies entirely in an open half-space of $W$. Using the previous argument, either $\left(\boldsymbol{\beta}^{1}\right)^{T} \overline{\boldsymbol{x}_{i}}<\left(\boldsymbol{\beta}^{2}\right)^{T} \overline{\boldsymbol{x}_{i}}$ for all $i \in S_{t}$, or $\left(\boldsymbol{\beta}^{1}\right)^{T} \overline{\boldsymbol{x}_{i}}>\left(\boldsymbol{\beta}^{2}\right)^{T} \overline{\boldsymbol{x}_{i}}$ for all $i \in S_{t}$.

Proposition 3.19. Generalized resistant hyperplane mechanisms are well defined. That is, given a family $\mathcal{S}=\left(S_{1}, \ldots, S_{d+1}\right)$ of publicly separable sets of agents, and $\boldsymbol{k}=\left(k_{1}, \ldots, k_{d+1}\right)$ with $k_{t} \in\left[\left|S_{t}\right|\right]$ for $t \in[d+1]$, there exists a unique hyperplane $\boldsymbol{\beta}$ for which $\min _{i \in S_{t}}^{k_{t}} y_{i}-\boldsymbol{\beta}^{T} \overline{\boldsymbol{x}_{i}}=0$ for each $t \in[d+1]$.

${ }^{7}$ While Theorem 3.14 uses $d+1$ well separable sets in $\mathbb{R}^{d+1}$, even $\mathbb{R}^{d}$ allows up to $d+1$ well separable sets. 
Proof. First, we show that if such a hyperplane exists, it must be unique. Suppose for contradiction that there are two distinct hyperplanes $\boldsymbol{\beta}^{1}$ and $\boldsymbol{\beta}^{2}$ which make the $k_{t}^{\text {th }}$ smallest residual from every $S_{t} \in \mathcal{S}$ zero. By the hyperplane comparison lemma (Lemma 3.18), there exists $S_{t} \in \mathcal{S}$ such that either $\left(\boldsymbol{\beta}^{1}\right)^{T} \overline{\boldsymbol{x}_{i}}<\left(\boldsymbol{\beta}^{2}\right)^{T} \overline{\boldsymbol{x}_{i}}$ for all $i \in S_{t}$, or $\left(\boldsymbol{\beta}^{1}\right)^{T} \overline{\boldsymbol{x}_{i}}>\left(\boldsymbol{\beta}^{2}\right)^{T} \overline{\boldsymbol{x}_{i}}$ for all $i \in S_{t}$. Without loss of generality, suppose it is the former. Then, at least $k_{t}$ points in $S_{t}$ which have a non-positive residual under $\boldsymbol{\beta}^{2}$ have a negative residual under $\boldsymbol{\beta}^{1}$, contradicting the fact that $\boldsymbol{\beta}^{1}$ makes the $k_{t}^{\text {th }}$ smallest residual from $S_{t}$ zero.

For proving existence, we use a counting technique. Create two bipartite graphs $G=(V \cup W, E)$ and $G^{\prime}=\left(V^{\prime} \cup W, E^{\prime}\right)$. Let $V\left(\right.$ resp. $\left.V^{\prime}\right)$ contain a vertex $v_{\boldsymbol{k}}\left(\right.$ resp. $\left.v_{\boldsymbol{k}}^{\prime}\right)$ corresponding to each $\boldsymbol{k}=\left(k_{1}, \ldots, k_{d+1}\right)$ such that $k_{t} \in\left[\left|S_{t}\right|\right]$ for each $t \in[d+1]$. Thus, $|V|=\left|V^{\prime}\right|=\prod_{t=1}^{d+1}\left|S_{t}\right|$. Let $W$ contain a vertex $w_{\beta}$ corresponding to every traversal hyperplane $\boldsymbol{\beta}$, i.e., every hyperplane that passes through at least one point from each set $S_{t} \in \mathcal{S}$.

In graph $G$, we draw an edge between $v_{k}$ and $w_{\beta}$ if $\boldsymbol{\beta}$ makes the $k_{t}^{\text {th }}$ smallest residual zero in each $S_{t} \in \mathcal{S}$. For constructing graph $G^{\prime}$, we fix an arbitrary ordering of points in each set, so that we can write $S_{t}=\left\{i_{1}^{t}, \ldots, i_{\left|S_{t}\right|}^{t}\right\}$. Then, we draw an edge in $G^{\prime}$ between $v_{\boldsymbol{k}}^{\prime}$ and $w_{\boldsymbol{\beta}}$ if $\boldsymbol{\beta}$ passes through point $i_{k_{t}}^{t}$ for each $t \in[d+1]$.

Our goal is to show that each vertex $v_{k} \in V$ has exactly one incident edge in graph $G$. We prove this through a sequence of claims. First, we argue that each vertex $v_{k}^{\prime} \in V^{\prime}$ has exactly one incident edge in graph $G^{\prime}$. The fact that it has at least one incident edge follows from the fact that any set of $d+1$ points in $\mathbb{R}^{d+1}$ (in particular, $T=\left\{i_{k_{t}}^{t}\right\}_{t \in[d+1]}$ ) lie on a hyperplane. If $\boldsymbol{v}_{\boldsymbol{k}}^{\prime}$ has two or more incident edges, then there exist two distinct hyperplanes $\boldsymbol{\beta}^{1}$ and $\boldsymbol{\beta}^{2}$ which pass through all points in $T$. Then, their intersection $\boldsymbol{\beta}^{*}$, which is a $(d-1)$-dimensional flat in $\mathbb{R}^{d+1}$, must also pass through all points in $T$. Let $\mathcal{P}(\boldsymbol{x}, \mathcal{S})=\left(X_{1}, \ldots, X_{d+1}\right)$. Then, the projection of $\boldsymbol{\beta}^{*}$ on the public information space $\mathbb{R}^{d}$ is a $(d-1)$-dimensional hyperplane in $\mathbb{R}^{d}$ which intersects each $X_{t}$ (and thus each $\left.\operatorname{Conv}\left(X_{t}\right)\right)$. However, $\mathcal{S}$ is a publicly separable family, i.e., $X_{1}, \ldots, X_{d+1}$ are well separable in $\mathbb{R}^{d}$. This violates the first condition of Proposition 3.12.

Since each vertex in $V^{\prime}$ has exactly one incident edge, we have $\left|E^{\prime}\right|=\left|V^{\prime}\right|=\prod_{t=1}^{d+1}\left|S_{t}\right|$. We next argue that $|E|=\left|E^{\prime}\right|$. Take a vertex $w_{\boldsymbol{\beta}} \in W$. Note that if hyperplane $\boldsymbol{\beta}$ passes through $a_{t}$ points from each $S_{t} \in \mathcal{S}$, then it has degree $\prod_{t=1}^{d+1} a_{t}$ in both $G$ and $G^{\prime}$. Since each vertex in $W$ has the same degree in both graphs, we have $|E|=\left|E^{\prime}\right|=\left|V^{\prime}\right|=|V|$.

Finally, we already established that if there is a hyperplane which makes the $k_{t}^{\text {th }}$ smallest residual in each $S_{t}$ zero, then it must be unique. Thus, each vertex in $V$ has at most one incident edge in $G$. Together with $|E|=|V|$, this implies that each vertex in $V$ has exactly one incident edge in $G$.

We are now ready to present our main contribution.

\section{THEOREM 3.20. Every generalized resistant hyperplane mechanism is group strategyproof.}

Proof. Consider an $(\mathcal{S}, \boldsymbol{k})$-generalized resistant hyperplane mechanism. Consider a set of data points $\mathcal{D}=\left(\boldsymbol{x}_{i}, y_{i}\right)_{i \in N}$. Suppose a coalition $S \subseteq N$ of agents changes their report to $\left(\widetilde{y}_{i}\right)_{i \in S}$, and changes the resulting hyperplane from $\boldsymbol{\beta}$ to $\widetilde{\boldsymbol{\beta}}$. Set $\widetilde{y}_{i}=y_{i}$ for $i \in N \backslash S$, and let $\widetilde{\mathcal{D}}=\left(\boldsymbol{x}_{i}, \widetilde{y}_{i}\right)_{i \in N}$.

By the hyperplane comparison lemma (Lemma 3.18), there exists $S_{t} \in \mathcal{S}$ such that either $\boldsymbol{\beta}^{T} \overline{\boldsymbol{x}_{i}}<\widetilde{\boldsymbol{\beta}}^{T} \overline{\boldsymbol{x}_{i}}$ for all $i \in S_{t}$, or $\boldsymbol{\beta}^{T} \overline{\boldsymbol{x}_{i}}>\widetilde{\boldsymbol{\beta}}^{T} \overline{\boldsymbol{x}_{i}}$ for all $i \in S_{t}$.

Without loss of generality, suppose it is the former. The $k_{t}^{\text {th }}$ smallest residual from $S_{t}$ is zero under $\boldsymbol{\beta}$ in $\mathcal{D}$, and under $\widetilde{\boldsymbol{\beta}}$ in $\widetilde{\mathcal{D}}$. If $S \cap S_{t}=\emptyset$, or if every manipulator in $S \cap S_{t}$ has a positive residual under $\boldsymbol{\beta}$ in $\mathcal{D}$, then at least $k_{t}$ non-manipulators in $N \backslash S$ have a non-positive residual under $\boldsymbol{\beta}$ in $\mathcal{D}$, and thus a strictly negative residual under $\widetilde{\boldsymbol{\beta}}$ in $\widetilde{\mathcal{D}}$, which contradicts the fact that $\widetilde{\boldsymbol{\beta}}$ makes the $k_{t}^{\text {th }}$ smallest residual in $S_{t}$ zero in $\widetilde{\mathcal{D}}$. 
In other words, there must exist a manipulator $i \in S \cap S_{t}$ who has a non-positive residual under $\boldsymbol{\beta}$ in $\mathcal{D}$. Thus, $\widetilde{\boldsymbol{\beta}}^{T} \overline{\boldsymbol{x}_{i}}>\boldsymbol{\beta}^{T} \overline{\boldsymbol{x}_{i}} \geq y_{i}$, implying that the manipulator is strictly worse off after the manipulation. Hence, the mechanism is group strategyproof.

For two dimensions $(d=1)$, we already argued that our sub-family of group strategyproof CRM mechanisms given by Theorem 3.7 is part of the larger family of GRL mechanisms (Lemma 3.10). It is easy to see that GRL mechanisms are precisely GRH mechanisms in two dimensions. Indeed, GRH mechanisms would require two subsets of agents $S_{1}, S_{2}$ that are publicly separable, i.e., well separable on the $x$-axis. Note that this coincides with the separability definition used by GRL mechanisms (Definition 3.6). Hence, the $\left(S, S^{\prime}, k, k^{\prime}\right)$-GRL mechanism is precisely the $(\mathcal{S}, \boldsymbol{k})$-GRH mechanism with $\mathcal{S}=\left(S, S^{\prime}\right)$ and $\boldsymbol{k}=\left(k, k^{\prime}\right)$. In three or more dimensions, we do not know if, given $\boldsymbol{x}$, one can always construct a family $\mathcal{S}$ of publicly separable sets of agents such that each set $S_{t} \in \mathcal{S}$ contains at least a constant fraction of the agents.

\subsection{Strategyproofness vs Group Strategyproofness}

In the single dimensional setting $(d=0)$, Moulin [33] proved that all strategyproof mechanisms are also group strategyproof. This alternatively follows from a result by Barberà et al. [2], who gave a sufficient condition on the underlying domain for the sets of strategyproof and group strategyproof mechanisms to coincide.

Interestingly, all known strategyproof mechanisms for the multidimensional linear regression setting (including generalized $L_{1}$-ERM and generalized resistant hyperplane mechanisms) are group strategyproof as well. However, it is easy to check that the linear regression setting does not satisfy the sufficient condition of Barberà et al. [2]. Is it still true that all strategyproof mechanisms for linear regression are also group strategyproof? We answer this question negatively.

Example 3.21. Consider the simple linear regression setting $(d=1)$ with $n=2$ agents. Fix the public information $\boldsymbol{x}=\left(x_{1}, x_{2}\right) \in \mathbb{R}^{2}$, and consider the mechanism $M$ that, on input $\boldsymbol{y}=\left(y_{1}, y_{2}\right)$, returns the line passing through points $\left(x_{1}, y_{2}\right)$ and $\left(x_{2}, y_{1}\right)$. Under this mechanism, the outcome for each agent is independent of the agent's report: indeed, the outcome for agent 1 (resp. agent 2 ) is $\widehat{y}_{1}=y_{2}$ (resp. $\widehat{y}_{2}=y_{1}$ ). Hence, the mechanism is clearly strategyproof. However, group strategyproofness is violated because when $y_{1} \neq y_{2}$, the two agents can collude, and report $\tilde{\boldsymbol{y}}=\left(y_{2}, y_{1}\right)$. This makes the resulting line pass through both agents, making both strictly better off.

The requirement that the outcome for each agent be independent of the agent's report, called impartiality in mechanism design, is stricter than (i.e., logically implies) strategyproofness, and has been studied for aggregating opinions or dividing rewards [14, 19, 22, 28, 39].

Definition 3.22 (Impartial Mechanisms). A mechanism $M$ is called impartial if the outcome for each agent is independent of the agent's report. Formally, for every agent $i \in N$, reports $\boldsymbol{y}$, and alternative report $y_{i}^{\prime}$ by agent $i$, we require that $\widehat{y}_{i}(M(\boldsymbol{y}))=\widehat{y}_{i}\left(M\left(y_{i}^{\prime}, \boldsymbol{y}_{-i}\right)\right)$.

In linear regression, when the number of agents is $n=d+1$, we can easily characterize all impartial mechanisms because we can set $\widehat{y}_{i}$ to be an arbitrary function of $\boldsymbol{y}_{-i}$, and return a hyperplane passing through the resulting $d+1$ points $\left(\boldsymbol{x}_{i}, \widehat{y}_{i}\right)_{i \in N}$.

Proposition 3.23. For $n=d+1$, mechanism $M$ is impartial if and only if there exist functions $f_{1}, \ldots, f_{n}: \mathbb{R}^{n-1} \rightarrow \mathbb{R}$ such that given $\boldsymbol{y}, M$ returns a hyperplane passing through $\left(\boldsymbol{x}_{i}, f_{i}\left(\boldsymbol{y}_{-i}\right)\right)_{i \in N}$

Note that functions $f_{i}$ can even be discontinuous, which can make the regression hyperplane discontinuous in the input $\boldsymbol{y}$. However, we later show (Theorem 4.3) that under any strategyproof mechanism, the outcome $\widehat{y}_{i}$ for agent $i$ must be a continuous function of $y_{i}$ (it is a constant function of $y_{i}$ in case of impartial mechanisms). 
With $n>d+1$ points, the question of whether impartial mechanisms even exist is non-trivial. While we still need to set each $\widehat{y}_{i}$ as a function of $\boldsymbol{y}_{-i}$, it cannot be done arbitrarily as the resulting points $\left(\boldsymbol{x}_{i}, \widehat{y}_{i}\right)_{i \in N}$ may no longer lie on a hyperplane. In other words, setting $\widehat{y}_{i}$ as a function of $\boldsymbol{y}_{-i}$ for $d+1$ agents already determines the hyperplane, and thus $\widehat{y}_{j}$ for all remaining agents $j$. The mechanism must ensure that these $\widehat{y}_{j}$ are also independent of $y_{j}$. At first glance, this may seem impossible, except in the trivial case where a constant hyperplane is returned regardless of $\boldsymbol{y}$.

Nonetheless, we show that there exists a wide family of non-trivial impartial mechanisms for linear regression. Our family provides a full characterization of impartial mechanisms for $d=1$ (i.e., for simple linear regression). In the result below, we use the notation $\langle\boldsymbol{a}, \boldsymbol{b}\rangle$ instead of $\boldsymbol{a}^{T} \boldsymbol{b}$ for the sake of simplicity. Its proof is in the full version.

TheOrem 3.24. Given $\boldsymbol{x}$, mechanism $M^{\boldsymbol{x}}$ for linear regression is impartial if there exist functions $\left\{g_{i}^{\boldsymbol{x}}: \mathbb{R} \rightarrow \mathbb{R}^{d}\right\}_{i \in N}$ and constant $c^{\boldsymbol{x}} \in \mathbb{R}$ such that for all $\boldsymbol{y}$, we have $M^{\boldsymbol{x}}(\boldsymbol{y})=\boldsymbol{\beta}=\left(\boldsymbol{\beta}_{1}, \beta_{0}\right)$, where

$$
\boldsymbol{\beta}_{1}=\sum_{i \in N} g_{i}^{\boldsymbol{x}}\left(y_{i}\right), \quad \beta_{0}=c^{\boldsymbol{x}}-\sum_{i \in N}\left\langle g_{i}^{\boldsymbol{x}}\left(y_{i}\right), \boldsymbol{x}_{i}\right\rangle .
$$

For $d=1$ and an admissible set of points, this characterizes all impartial mechanisms.

Impartial mechanisms are not compelling from a statistical viewpoint. For instance, in the standard two-dimensional stochastic model where the data points are assumed to be generated by taking points on an underlying line and introducing i.i.d. errors in the dependent variables, it is easy to show that no impartial mechanism can produce an unbiased estimator of the underlying line. Nonetheless, impartial mechanisms help us establish the existence of a rather wide family of strategyproof mechanisms that are not group strategyproof. In fact, the next result shows that almost all impartial mechanisms violate group strategyproofness; its proof is in the full version.

Proposition 3.25. For simple linear regression $(d=1)$ with an admissible set of points, an impartial mechanism is group strategyproof if and only if it is a constant function (i.e., it returns a fixed regression line regardless of its input).

\section{CHARACTERIZING STRATEGYPROOF MECHANISMS}

As mentioned in Section 3.1, Moulin [33] studied the one-dimensional setting $(d=0)$, and analytically characterized all strategyproof mechanisms for $n$ agents. While we are unable to provide an analytical characterization for multidimensional linear regression, we provide two non-constructive characterizations, and discuss their implications.

Interestingly, to characterize strategyproof mechanisms for linear regression with $n$ agents, we use the characterization of strategyproof mechanisms for the one-dimensional setting with a single agent. In this case, Moulin [33] shows that a mechanism is strategyproof if and only if there exist constants $\alpha^{1}, \alpha^{2} \in \overline{\mathbb{R}}$ such that when the agent reports $y$, the mechanism returns $\widehat{y}=\operatorname{med}\left(y, \alpha^{1}, \alpha^{2}\right)$. Constants $\alpha^{1}$ and $\alpha^{2}$ are called phantoms. First, we extend this result by providing an alternative characterization, which uses the following definition. The proof of the next result is in the full version.

Definition 4.1 (Locally Constant Function). For $A, B \subseteq \mathbb{R}$, function $f: A \rightarrow B$ is called locally constant at $x \in A$ if there exists $\epsilon>0$ such that for all $x^{\prime} \in[x-\epsilon, x+\epsilon], f\left(x^{\prime}\right)=f(x)$.

Lemma 4.2. Suppose mechanism $\pi: \mathbb{R} \rightarrow \mathbb{R}$ for the one-dimensional setting with a single agent elicits private value $y$ from the agent and returns $\pi(y)$. Then, $\pi$ being strategyproof is equivalent to each of the following conditions.

(a) There exist constants $\alpha^{1}, \alpha^{2} \in \overline{\mathbb{R}} \triangleq \mathbb{R} \cup\{-\infty, \infty\}$ such that for all $y \in \mathbb{R}, \pi(y)=\operatorname{med}\left(y, \alpha^{1}, \alpha^{2}\right)$.

(b) $\pi$ is continuous, and for every $y \in \mathbb{R}$, either $\pi(y)=y$ or $\pi$ is locally constant at $y$. 
In the one-dimensional setting, Moulin [33] observed that a mechanism is strategyproof if and only if its outcome is strategyproof in the report of each individual agent when other agents' reports are fixed. That is, a mechanism $\pi: \mathbb{R}^{n} \rightarrow \mathbb{R}$ for $n$ agents is strategyproof if and only if

$$
\forall i \in[n], \exists \alpha_{i}^{1}, \alpha_{i}^{2} \in \overline{\mathbb{R}} \text { independent of } y_{i} \text { s.t. } \pi\left(y_{1}, \ldots, y_{n}\right)=\operatorname{med}\left(y_{i}, \alpha_{i}^{1}, \alpha_{i}^{2}\right) .
$$

Moulin [33] solved Equation (7) to derive an elegant analytical expression for $\pi$ in terms of $\left\{y_{i}\right\}_{i \in[n]}$. Note that in this equation, the outcome $\widehat{y}=\pi\left(y_{1}, \ldots, y_{n}\right)$ is common to all agents.

In contrast, in linear regression each agent $i$ has a potentially different outcome $\widehat{y}_{i}$. Like before, strategyproofness requires that each $\widehat{y}_{i}$ obey the conditions in Lemma 4.2, when seen as a function of $y_{i}$, when other agents' reports are fixed. However, the outcomes for different agents are now constrained so that $\left(\boldsymbol{x}_{i}, \widehat{y}_{i}\right)_{i \in N}$ lie on a hyperplane. This added complexity prevented us from solving the equations to derive an analytical characterization, despite significant effort. The only exception was the special case of impartial mechanisms, where we further restrict $\widehat{y}_{i}$ to be independent of $y_{i}$ (Theorem 3.24). This corresponds to the case where $\alpha_{i}^{1}=\alpha_{i}^{2}$ for each agent $i$. Nonetheless, by simply applying Lemma 4.2 for every agent $i$, we obtain the following non-constructive characterization of strategyproof mechanisms for linear regression.

Theorem 4.3. Given public information $\boldsymbol{x}$, mechanism $M^{\boldsymbol{x}}$ for linear regression being strategyproof is equivalent to each of the following conditions.

(a) For every $\boldsymbol{y}_{-i} \in \mathbb{R}^{n-1}$ and $i \in N$, there exist $\ell_{i}, h_{i} \in \overline{\mathbb{R}}$ such that $\widehat{y}_{i}\left(M^{x}(\boldsymbol{y})\right)=\operatorname{med}\left(y_{i}, \ell_{i}, h_{i}\right)$ for all $y_{i} \in \mathbb{R}$;

(b) For every $\boldsymbol{y}_{-i} \in \mathbb{R}^{n-1}$ and $i \in N$, function $f_{i}(\cdot)=\widehat{y}_{i}\left(M\left(\cdot, \boldsymbol{y}_{-i}\right)\right)$ is continuous, and for every $y_{i} \in \mathbb{R}$, either $f_{i}\left(y_{i}\right)=y_{i}$ or $f_{i}$ is locally constant at $y_{i}$.

The first condition provides an analytical form of $\widehat{y}_{i}$ in terms of $y_{i}$, and is perhaps the more useful characterization. For instance, we crucially use this characterization in the next section to give a lower bound on the efficiency of strategyproof mechanisms. Our earlier (more complex) proof of group strategyproofness of GRH mechanisms (Theorem 3.20) was also based on this condition, and identified the precise $\ell_{i}$ and $h_{i}$ for each agent $i$.

Note that for fixed $\boldsymbol{y}_{-i}$, we have $\widehat{y}_{i}=y_{i}$ when $y_{i} \in\left[\ell_{i}, h_{i}\right]$. For $y_{i} \leq \ell_{i}, \widehat{y}_{i}=\ell_{i}$ is fixed, and for $y_{i} \geq h_{i}, \widehat{y}_{i}=h_{i}$ is fixed. We therefore say that agent $i$ is influential over the interval $\left(\ell_{i}, h_{i}\right)$, and call $\ell_{i}$ and $h_{i}$ the lower and upper influence bounds, respectively. Analysis of influence bounds has received attention in the statistics literature, where it is called sensitivity analysis. For instance, Narula and Wellington [34] observed that under $L_{1}$-ERM, the regression hyperplane is unaffected when the dependent variable of a point is changed so that the point still lies on the same side of the hyperplane as before. From Theorem 4.3, we can see that for every strategyproof mechanism, doing so should at least keep the outcome for agent $i$ unchanged. Narula and Wellington [34] also focused on computing the influence bounds. Theorem 4.3 lends a simple algorithm to compute influence bounds (see the full version). Finally, note that while $\widehat{y}_{i}$ must be continuous in $y_{i}$, it need not be continuous in $\boldsymbol{y}$ (see our discussion on Proposition 3.23).

\section{EFFICIENCY OF STRATEGYPROOF MECHANISMS}

Insofar, we studied families of strategyproof mechanisms for linear regression. In the absence of strategic considerations, a popular mechanism for linear regression is the OLS (ordinary least squares), which is the empirical risk minimizer for the squared loss. Under this loss function, which is also called the residual sum of squares (RSS), the loss when choosing hyperplane $\boldsymbol{\beta}$ given data points $\mathcal{D}$ is $\operatorname{RSS}(\mathcal{D}, \boldsymbol{\beta})=\sum_{i \in N}\left(y_{i}-\boldsymbol{\beta}^{T} \overline{\boldsymbol{x}_{i}}\right)^{2}$. A classic justification for the OLS is due to the Gauss-Markov theorem, which states that when the errors (deviations of data points from an 
underlying hyperplane we wish to identify) are stochastic, zero in expectation, uncorrelated, and of equal variance, the OLS is the best linear unbiased estimator.

However, in our strategic setting, the OLS is not strategyproof [15]. This raises an important question: Is there a strategyproof mechanism that is close to the OLS? We assess this by the worst-case approximation ratio of a mechanism for the optimal squared loss.

Definition 5.1 (Efficiency). Given $\boldsymbol{x}$, we say that mechanism $M^{x}$ for linear regression is $c$-efficient if for every $\mathcal{D}=\left(\boldsymbol{x}_{i}, y_{i}\right)_{i \in N}$, we have $\operatorname{RSS}\left(\mathcal{D}, M^{x}(\boldsymbol{y})\right) \leq c \cdot \inf _{\boldsymbol{\beta}} \operatorname{RSS}(\mathcal{D}, \boldsymbol{\beta})$.

We show that no strategyproof mechanism that is too close to the OLS can be strategyproof. The proof of the next result leverages our characterization of strategyproof mechanisms (Theorem 4.3).

Theorem 5.2. For $n \geq 4$, there exist $\boldsymbol{x}$ for which no strategyproof mechanism is $(2-\epsilon)$-efficient for any $\epsilon>0$.

Proof. For simplicity of notation, we use $n+1$ agents instead of $n$ agents (and assume $n+1 \geq 4$, i.e., $n \geq 3)$. We also consider simple linear regression $(d=1)$; the proof easily extends to higher dimensions by simply setting all other coordinates to zero. Fix $n \geq 3$. Consider a setting with $n+1$ agents where $x_{i}=i$ for $i \in[n]$, and $x_{n+1}=X$, where $X$ is the solution of the following equation:

$$
\frac{n^{3}-n}{2\left(1+3 n+2 n^{2}+6 X^{2}-6 X n-6 X\right)}=1 .
$$

Interested readers may note that $X=\Theta\left(n^{1.5}\right)$. Let $T$ denote the LHS in Equation (8).

Consider a strategyproof mechanism $M^{x}$. Suppose $M^{x}$ is $c$-efficient. We want to show that $c \geq 2$. We consider a family of inputs $\boldsymbol{y}$, in which we fix $y_{i}=0$ for $i \in[n]$, and vary $y_{n+1}=Y$. First, we note that the optimal RSS, as a function of $Y$, is given by

$$
f_{0}(Y)=Y^{2} \cdot \frac{n^{3}-n}{2+5 n+4 n^{2}+n^{3}-12 X-12 n X+12 X^{2}}=Y^{2} \cdot \frac{T}{T+1}=\frac{Y^{2}}{2},
$$

where the first transition is obtained by minimizing $\left(Y-X \cdot \beta_{1}-\beta_{0}\right)^{2}+\sum_{i=1}^{n}\left(i \cdot \beta_{1}+\beta_{0}\right)^{2}$ over all $\left(\beta_{1}, \beta_{0}\right)$, the second transition follows through simple algebra, and the final transition follows from Equation (8).

Recall that we fixed $y_{i}$ for $i \in[n]$. Due to our characterization result (Theorem 4.3), there exist $\ell, h \in \overline{\mathbb{R}}$ with $\ell \leq h$ such that the line returned by the mechanism passes through $(X, \operatorname{med}(Y, \ell, h))$ for all $Y$. We take two cases.

Case 1: $h>0$. Set $Y=h$. Then, the line returned by the mechanism passes through $(X, h)$. In this case, we can show that the RSS of the mechanism is at least

$$
f_{1}=h^{2} \cdot \frac{n^{3}-n}{2\left(1+3 n+2 n^{2}+6 X^{2}-6 X n-6 X\right)}=h^{2} \cdot T=h^{2},
$$

where the first transition is obtained by minimizing $\left(Y-\beta_{1} \cdot X-\beta_{0}\right)^{2}+\sum_{i=1}^{n}\left(\beta_{1} \cdot i+\beta_{0}\right)^{2}$ over all $\left(\beta_{1}, \beta_{0}\right)$ which satisfy $\beta_{1} \cdot X+\beta_{0}=Y$, and the rest follows from Equation (8). This implies $c \geq f_{1} / f_{0}(h)=2$.

Case 2: $h \leq 0$. Set $Y=1$. Then, the line returned by the mechanism passes through $(X, h)$. In this case, the RSS of the mechanism is at least $f_{2}=1$ because agent $n+1$ contributes $(1-h)^{2} \geq 1$ to the squared loss. Once again, we have $c \geq f_{2} / f_{0}(1)=2$.

The proof is complete as we have $c \geq 2$ in each case.

For $n=2$ agents (or $n=d+1$ agents in $d+1$ dimensions), there is an obvious 1-efficient strategyproof mechanism which returns a hyperplane passing through all input points. Theorem 4.3 leaves open the case of $n=3$ in two dimensions. 


\section{DISCUSSION}

Our work leaves several open questions. Perhaps the most ambitious one is to find a constructive characterization of all strategyproof or group strategyproof mechanisms for linear regression, which may allow us to pinpoint the most efficient strategyproof mechanism; Caragiannis et al. [8] provide a similar analysis in the one-dimensional setting. It is easy to show that $L_{1}$-ERM is $n$-efficient (see the full version). Does there exist a more efficient strategyproof mechanism? It would also be interesting to analyze efficiency in a stochastic setting where the data points are drawn from an underlying distribution.

The characterization result of Moulin [33] for strategyproof and anonymous mechanisms in the one-dimensional setting extends the median to generalized medians by adding fixed phantom values, and then taking the median. It is also shown that adding $n+1$ phantoms is sufficient to obtain full generality. We can extend all our proposed families of mechanisms by adding a certain number of "phantom points" in $\mathbb{R}^{d+1}$, and then applying the mechanisms to the union of data points and phantom points. The resulting mechanism retains the incentive guarantees. ${ }^{8}$ Given $n$ data points, how many phantoms are sufficient to obtain full generality? Do the phantoms play a role in obtaining the elusive constructive characterization?

Another interesting observation is that our generalized resistant hyperplane mechanisms are guaranteed pass through $d+1$ input points in $d+1$ dimensions. It is known that at least one minimizer of the $L 1$ loss also has this property. It would be interesting to identify a generic family of conditions, which, when imposed in addition to the requirement of making $d+1$ residuals zero, yield group strategyproofness.

Finally, Dekel et al. [15] study a regression setting in which a single agent may control multiple data points, show that $L_{1}$-ERM is no longer strategyproof, and provide novel strategyproof mechanisms. It would be useful to see if our ideas can be used to design additional strategyproof mechanisms in this model. Another interesting variant is when only a small number of data points are held by strategic agents, but the mechanism does not know which ones. A similar setting was studied by Charikar et al. [11], but for classification and with adversarial manipulations. On a high level, we view our work as a stepping stone to studying incentives in more realistic machine learning environments.

\section{REFERENCES}

[1] I. Bárány, A. Hubard, and J. Jerónimo. 2008. Slicing convex sets and measures by a hyperplane. Discrete \& Computational Geometry 39, 1-3 (2008), 67-75.

[2] S. Barberà, D. Berga, and B. Moreno. 2010. Individual versus group strategy-proofness: When do they coincide? fournal of Economic Theory 145, 5 (2010), 1648-1674.

[3] D. Black. 1958. Theory of Committees and Elections. Cambridge University Press.

[4] F. Breuer. 2010. Uneven splitting of ham sandwiches. Discrete \& Computational Geometry 43, 4 (2010), 876-892.

[5] G. W. Brown and A. M. Mood. 1951. On Median Tests for Linear Hypotheses. In Proceedings of the 2nd Berkeley Symposium on Mathematical Statistics and Probability. 159-166.

[6] N. H. Bshouty, N. Eiron, and E. Kushilevitz. 2002. PAC Learning with Nasty Noise. Theoretical Computer Science 288, 2 (2002), 255-275.

[7] Y. Cai, C. Daskalakis, and C. H. Papadimitriou. 2015. Optimum Statistical Estimation with Strategic Data Sources. In Proceedings of the 28th Conference on Computational Learning Theory (COLT). 280-296.

[8] I. Caragiannis, A. D. Procaccia, and N. Shah. 2016. Truthful Univariate Estimators. In Proceedings of the 33rd International Conference on Machine Learning (ICML). 127-135.

[9] F. Caro and J. Gallien. 2010. Inventory Management of a Fast-Fashion Retail Network. Operations Research 58, 2 (2010), 257-273.

\footnotetext{
${ }^{8} \mathrm{We}$ also considered adding phantom values directly in the equations where a median is used. However, most such attempts violated strategyproofness.
} 
[10] F. Caro, J. Gallien, M. D. Miranda, J. C. Torralbo, J. M. C. Corras, M. M. Vazquez, J. A. R. Calamonte, and J. Correa. 2010. Zara Uses Operations Research to Reengineer its Global Distribution Process. Interfaces 40, 1 (2010), 71-84.

[11] M. Charikar, J. Steinhardt, and G. Valiant. 2017. Learning from untrusted data. In Proceedings of the 49th Annual ACM Symposium on Theory of Computing (STOC). 47-60.

[12] Yudong Chen, Constantine Caramanis, and Shie Mannor. 2013. Robust sparse regression under adversarial corruption. In International Conference on Machine Learning. 774-782.

[13] R. Cummings, S. Ioannidis, and K. Ligett. 2015. Truthful Linear Regression. In Proceedings of the 28th Conference on Computational Learning Theory (COLT). 448âĂŞ-483.

[14] G. de Clippel, H. Moulin, and N. Tideman. 2008. Impartial division of a dollar. Fournal of Economic Theory 139 (2008), 176-191.

[15] O. Dekel, F. Fischer, and A. D. Procaccia. 2010. Incentive Compatible Regression Learning. F. Comput. System Sci. 76, 8 (2010), 759-777.

[16] J. Dong, A. Roth, Z. Schutzman, B. Waggoner, and Z. S. Wu. 2017. Strategic Classification from Revealed Preferences. arXiv:1710.07887. (2017).

[17] M. Dummett and R. Farquharson. 1961. Stability in voting. Econometrica 29, 1 (1961), 33-43.

[18] J. H. Elton and T. P. Hill. 2011. A stronger conclusion to the classical ham sandwich theorem. European fournal of Combinatorics 32, 5 (2011), 657-661.

[19] F. Fischer and M. Klimm. 2015. Optimal impartial selection. SIAM J. Comput. 44, 5 (2015), 1263-1285.

[20] S. A. Goldman and R. H. Sloan. 1995. Can PAC Learning Algorithms Tolerate Random Attribute Noise? Algorithmica 14, 1 (1995), 70-84.

[21] M. Hardt, N. Megiddo, C. H. Papadimitriou, and M. Wootters. 2016. Strategic Classification. In Proceedings of the 7th Innovations in Theoretical Computer Science Conference (ITCS). 111-122.

[22] R. Holzman and H. Moulin. 2013. Impartial nominations for a prize. Econometrica 81, 1 (2013), 173-196.

[23] S. Ioannidis and P. Loiseau. 2013. Linear regression as a non-cooperative game. In Proceedings of the 9th Conference on Web and Internet Economics (WINE). 277-290.

[24] I. M. Johnstone and P. F. Velleman. 1985. The resistant line and related regression methods. F. Amer. Statist. Assoc. 80, 392 (1985), 1041-1054.

[25] M. Kearns and M. Li. 1993. Learning in the Presence of Malicious Errors. SIAM f. Comput. 22, 4 (1993), 807-837.

[26] H. Kermer and A. B. Németh. 1973. Supporting spheres for families of independent convex sets. Archiv der Mathematik 24, 1 (1973), 91-96.

[27] R. Koenker and Gilbert Bassett, Jr. 1978. Regression quantiles. Econometrica 46, 1 (1978), 33-50.

[28] D. Kurokawa, O. Lev, J. Morgenstern, and A. D. Procaccia. 2015. Impartial Peer Review.. In Proceedings of the 24th International foint Conference on Artificial Intelligence (IfCAI). 582-588.

[29] N. Littlestone. 1991. Redundant noisy attributes, attribute errors, and linear-threshold learning using winnow. In Proceedings of the 4th Conference on Computational Learning Theory (COLT). 147-156.

[30] R. Meir, S. Almagor, A. Michaely, and J. S. Rosenschein. 2011. Tight bounds for strategyproof classification. In Proceedings of the 10th International Conference on Autonomous Agents and Multi-Agent Systems (AAMAS). 319-326.

[31] R. Meir, A. D. Procaccia, and J. S. Rosenschein. 2010. On the limits of dictatorial classification. In Proceedings of the 9th International Conference on Autonomous Agents and Multi-Agent Systems (AAMAS). 609-616.

[32] R. Meir, A. D. Procaccia, and J. S. Rosenschein. 2012. Algorithms for Strategyproof Classification. Artificial Intelligence 186 (2012), 123-156.

[33] H. Moulin. 1980. On strategy-proofness and single-peakedness. Public Choice 35 (1980), 437-455.

[34] S. C. Narula and J. F. Wellington. 1985. Interior analysis for the minimum sum of absolute errors regression. Technometrics 27, 2 (1985), 181-188.

[35] J. Perote and J. Perote-Peña. 2003. The impossibility of strategy-proof clustering. Economics Bulletin 4, 23 (2003), 1-9.

[36] J. Perote and J. Perote-Peña. 2004. Strategy-proof estimators for simple regression. Mathematical Social Sciences 47 (2004), 153-176.

[37] W. Steiger and J. Zhao. 2010. Generalized ham-sandwich cuts. Discrete \& Computational Geometry 44, 3 (2010), $535-545$.

[38] A. H. Stone and J. W. Tukey. 1942. Generalized "sandwich" theorems. Duke Mathematical fournal 9, 2 (1942), 356-359.

[39] S. Tamura and S. Ohseto. 2014. Impartial nomination correspondences. Social Choice and Welfare 43 (2014), 47-54.

[40] J. W. Tukey. 1977. Exploratory Data Analysis. Addison-Wesley.

[41] L. Wang. 2013. The L1 penalized LAD estimator for high dimensional linear regression. fournal of Multivariate Analysis 120 (2013), 135-151.

[42] L. Wang, M. D. Gordon, and J. Zhu. 2006. Regularized least absolute deviations regression and an efficient algorithm for parameter tuning. In Proceedings of the 6th IEEE International Conference on Data Mining (ICDM). 690-700. 